\title{
DELINEATION OF ANISOTROPIC LAYERS THROUGH 1D INVERSION OF MARINE CSEM DATA
}

\author{
Cícero R. Teixeira Régis ${ }^{1,2}$, Edelson da Cruz Luz ${ }^{1,3}$ and Walleson Gomes dos Santos ${ }^{1}$
}

\begin{abstract}
This paper describes a method to invert marine CSEM data from anisotropic layered media. The method uses two types of constraints to generate stable solutions that improve the positioning of interfaces in anisotropic layers: applying the $L_{1}$ norm equality constraints of the Total Variation method to parameters in adjacent layers, and imposing $\mathrm{L}_{2}$ norm equality constraints between the different components of the conductivity tensor in each layer. The solutions are compared favorably with those from previously published smoothing methods. The results show that the simultaneous application of the two constraints is able to improve the delineation of the anisotropic layers, including the resistive target commonly sought in the inversion of marine CSEM data. As an example, the method was applied to real data from an offshore Brazilian basin.
\end{abstract}

Keywords: anisotropic layered media, inversion, CSEM.

RESUMO. Este artigo descreve um método para inverter dados do método marinho de fonte controlada CSEM de meios anisotrópicos estratificados. 0 método usa dois tipos de vínculos para gerar soluções estáveis que melhoram o posicionamento de interfaces entre camadas anisotrópicas: aplicando os vínculos de norma $L_{1}$ do método de Variação Total a parâmetros de camadas adjacentes, e impondo vínculos de igualdade de norma $\mathrm{L}_{2}$ entre as componentes do tensor de impedância em cada camada. As soluções obtidas mostraram-se melhores do que as soluções encontradas na literatura com apenas o método de suavidade. Os resultados indicam que a aplicação simultânea dos dois vínculos é capaz de melhorar o delineamento de camadas anisotrópicas, incluindo as camadas-alvo resistivas normalmente buscadas na inversão de dados CSEM. 0 método foi aplicado a um conjunto de dados reais de uma bacia marinha brasileira.

Palavras-chave: meios estratificados anisotrópicos, inversão, CSEM.

\footnotetext{
1 Universidade Federal do Pará, Graduate Program in Geophysics, R. Augusto Correa, 1, 66075-110 Belém, PA, Brazil. Phone: +55(91) 3201-7692

_E-mails: cicero@ufpa.br; edelson.luz@ifpa.br; walleson_santos@yahoo.com.br

2Universidade Federal da Bahia, Instituto de Geociências, National Institute of Science and Technology of Petroleum Geophysics, sala 312-C, Campus de Ondina, 40170-115 Salvador, BA, Brazil.

3IFPA, Campus Marabá Rural, BR-155, km 25, s/n, Assentamento 26 de Março, Zona Rural, 68501-000 Marabá, PA, Brazil. Phone: +55(94) $99212-2642$
} 


\section{INTRODUCTION}

Inversion of marine CSEM data is a challenging task, because of the inherently low resolution in the data. The use of extremely low frequencies (Key, 2009), in a diffusive regime, contributes to this low resolution. This implies a high level of ambiguity in the data and generally low sensitivities. The situation is worse when anisotropy exists in the electrical conductivity of the media, because not only the ambiguity is increased but also the inversion seeks to extract even more information from the data than when isotropy is assumed.

Usually, the data misfit function includes the difference between the estimated and the observed data, and this difference is normalized by an estimate of the uncertainty of each observation, based on the standard deviation of the data error (Tarantola, 2005). This normalization weighs the influence of each observation on the misfit according to their uncertainty (Key, 2009).

Fitting marine CSEM data is difficult for any inversion algorithm because the fields show a very wide dynamic range, spanning several orders of magnitude, depending on the sourcereceiver offset. In such cases, in addition to weighing by the data uncertainty, a common practice is defining the data misfit function with the logarithms of the electric field amplitudes, which drastically shortens its possible range of values (Crepaldi et al., 2011). In this work, we use the misfit function suggested by Almeida \& Rijo (2011), which employs the normalization of each estimated data fit by the corresponding observation at the same spatial position. This measure of the relative difference between estimated and observed data leads to a well balanced data set, meaning that, in principle, if all observations had the same level of uncertainty they would all have the same level of influence on the misfit.

A popular method to generate stable solutions in geophysical inversion is to apply global smoothness constraints to the inversion parameters, like in the ubiquitous Occam's Inversion algorithm (Constable et al., 1987). This method introduces constraints which enforce equality between pairs of spatially adjacent parameters in a least squares sense, and it leads to smooth solutions. Traditionally, the $L_{2}$ norm is used to measure how well the model structures conform to the imposed constraints, but that is not the only possibility, as demonstrated by Farquharson \& Oldenburg (1998), who presented an extensive analysis of the effects of using general measures of misfit (of data, as well as of model adjustment) in the inversion of electromagnetic data. Total Variation regularization, developed in the area of image processing (Rudin et al., 1992; Vogel \& Oman, 1998), has been applied in the inversion of geophysical data (Martins et al., 2011; Lima et al., 2011) to estimate non-smooth basement relief in a sedi- mentary basin from gravity data. The method minimizes the differences between pairs of adjacent parameters in a $L_{1}$ norm misfit estimator. The sum of the absolute values of these differences is the discrete equivalent of the mathematical concept of the total variation of a function (Acar \& Vogel, 1994). The method allows for sharp variations to account for discontinuities in the distribution of the physical properties.

Application of the Total Variation method to electromagnetic data inversion has been presented by Schwarzbach \& Haber (2013), who performed a study with 3D synthetic data, but didn't find significant advantages in using this kind of constraint. In our application to 1D layered earth models, however, a stronger influence of the sharp discontinuities in the models is perceived by the data than in the case of $3 \mathrm{D}$ models, which is determinant to the success of the method. As we'll see in our results, in the worst case the Total Variation constraints lead to solutions that are equivalent to the ones from traditional smoothness methods.

Examples of $1 \mathrm{D}$ inversion of marine CSEM data from anisotropic layers were studied in Ramananjaona et al. (2011), with a method based on Occam regularization, with the expected smooth results. However, in many geological environments there can exist strong discontinuities in the physical properties of the media. In such cases, the results obtained by enforcing global smoothness, exclusively, in least square estimators can be misleading.

In this paper we present an approach to the inversion of marine CSEM data which addresses the problems of fitting the data, tackling the difficulty of resolving anisotropic conductivities and allowing non-smooth solutions. The inversion scheme implements a Gauss-Newton minimization with iterations of the Levenberg-Marquardt algorithm (Marquardt, 1963), with the following specifications, which are explained in detail in the following sections.

- The data misfit function is defined as the relative difference between the estimated and the observed data (Almeida \& Rijo, 2011).

- Equality constraints are imposed between the horizontal and vertical conductivities inside each anisotropic layer to control the degree of anisotropy imposed to the solutions.

- Total Variation regularization constraints are applied to the conductivities of adjacent layers (Lima et al., 2011; Martins et al., 2011; Schwarzbach \& Haber, 2013).

The application of these techniques resulted in a stable and meaningful solution to the problem of inverting marine CSEM data 
from anisotropic $1 \mathrm{D}$ models. In comparison with the application of global smoothness methods, like the Occam algorithm, this solution resulted in an improved resolution of the anisotropic layers, including the resistive target layers usually sought in hydrocarbon exploration. In particular, the results presented here for inversion of CSEM data exclusively (without adding magnetotelluric data nor a priori determination of any interfaces) compare quite favourably with those presented in Ramananjaona et al. (2011).

The simultaneous application of the two constraints has a true synergetic effect, allowing the method to achieve a good delineation of the target layer, meaning that it improves the identification of layer interfaces in comparison with smooth solutions. This delineation is a much more difficult task than the detection or location achieved by simply enforcing smooth solutions, as has been seen in previous works.

\section{MODELING}

To model the marine CSEM data, we calculate the electromagnetic field components from the electric dipole source in transversely isotropic layered media with a vertical symmetry axis (TIV), in the frequency domain. In this problem, the anisotropic conductivity is constant throughout each layer. The detailed derivation and analysis of the CSEM fields in anisotropic layered media is found in Santos \& Régis (2015).

Start with the frequency domain Maxwell's equations, using quasi-static approximation, in a conductive environment:

$$
\begin{aligned}
\nabla \times H-\sigma E & =I(\omega) d s \delta(x) \delta(y) \delta(z) . \\
\nabla \times E+i \omega \mu H & =0, \\
\nabla \cdot H & =0, \\
\nabla \cdot E & =0 .
\end{aligned}
$$

The term $I(\omega) d s \delta(x) \delta(y) \delta(z)$ represents the current density function of the dipole source, using Dirac delta functions. For an $x$ oriented dipole, $d s=\left(d s_{x}, 0,0\right)$. Then $I(\omega) d s_{x}$ represents the dipole moment.

The conductivity tensor is

$$
\sigma=\left(\begin{array}{ccc}
\sigma_{h} & 0 & 0 \\
0 & \sigma_{h} & 0 \\
0 & 0 & \sigma_{v}
\end{array}\right)
$$

Define the vector potential $\mathbf{A}$, so that

$$
H=\nabla \times A .
$$

Then, from Faraday law (Eq. 2):

$$
\begin{array}{r}
\nabla \times(E+i \omega \mu A)=0 ; \\
E=-i \omega \mu A-\nabla U,
\end{array}
$$

where $U$ is a scalar field.

Following the procedure described in Kaufman \& Dashevsky (2003), a convenient configuration for the vector potential is $A=$ $\left(A_{x}, 0, A_{z}\right)$. When combining Equations (8) and (1), the gauge condition

$$
\nabla \cdot A=-\sigma_{h} \cdot U
$$

allows us to write the electric field as

$$
E=-i \omega \mu A+\frac{1}{\sigma_{h}} \nabla(\nabla \cdot A) .
$$

The choice of this gauge condition leads to a system of two coupled differential equations on the components of $A$ :

$$
\begin{array}{r}
\nabla^{2} A_{x}+k_{h}^{2} A_{x}=-I(\omega) d s_{x} \delta(x) \delta(y) \delta(z), \\
\nabla^{2} A_{z}+k_{v}^{2} A_{z}=\left(1-\frac{\sigma_{v}}{\sigma_{h}}\right) \frac{\partial}{\partial z} \nabla \cdot A .
\end{array}
$$

Two wave numbers are defined: one associated with the horizontal conductivity $k_{h}^{2}=-i \omega \mu \sigma_{h}$, and the other associated with the vertical conductivity $k_{v}^{2}=-i \omega \mu \sigma_{v}$.

These equation are solved in the Fourier domain $\left(k_{x}, k_{y}, z\right)$, where $k_{x}$ and $k_{y}$ are the spectral coordinates corresponding to the spatial coordinates $x$ and $y$. The equations are valid inside each layer, and the solutions are linked via continuity conditions on the interfaces between layers. In this way, the solution is achieved as an infinite sequence of plane wave solutions in $z$. The components of the electric field are calculated by applying Eq. (10), so we include the necessary derivatives on the solutions in the Fourier transform. To achieve the final solution in $(x, y, z)$ we transform the double integral of the Fourier transform of the function with argument $\sqrt{k_{x}^{2}+k_{y}^{2}}$ into the single integral of the Hankel transform, with $k_{r}^{2}=k_{x}^{2}+k_{y}^{2}$ and $r^{2}=x^{2}+y^{2}$ (Arfken, 1985, p. 796):

$$
\begin{gathered}
\frac{1}{2 \pi} \int_{-\infty}^{\infty} \int_{-\infty}^{\infty} f\left(k_{x}^{2}+k_{y}^{2}\right) e^{i\left(k_{x} x+k_{y} y\right)} d k_{x} d k_{y} \\
=\int_{0}^{\infty} f\left(k_{r}\right) J_{0}\left(k_{r} r\right) k_{r} d k_{r} .
\end{gathered}
$$

In our problem, $f\left(k_{x}^{2}+k_{y}^{2}\right)$ is a frequency dependent function that carries the information about the source and the model, in the transform domain, including all reflection and transmission coefficients for the interfaces. The result is a set of two Hankel integrals for each field component, which are solved numerically, using the Fast Hankel transform filter presented in Anderson (1979). 


\section{INVERSION}

The set of parameters forms the vector $\mathbf{m}$. In this $1 \mathrm{D}$ problem, for $N$ layers there are $2 N$ parameters, which are the logarithms of the horizontal and vertical components of the conductivities $\left(\log _{10} \sigma_{h}\right.$ and $\left.\log _{10} \sigma_{v}\right)$. The function to be minimized is

$$
\Phi(\mathbf{m})=\phi^{d}(\mathbf{m})+\phi^{p}(\mathbf{m}),
$$

in which $\phi^{d}$ represents the misfit between calculated and observed data, and $\phi^{p}(\mathbf{m})$ is the functional used to introduce stabilizing constraints to the inversion parameters. Each of these functions perform a crucial role in the inversion process and both should influence the outcome. The estimated model responses must fit the observed data, but this requirement alone is not enough to guarantee a stable solution. To reach this goal, we impose that the function $\phi^{p}$ forces the solution to exhibit a desired attribute of the true source.

Functions $\phi^{d}$ and $\phi^{p}$ are defined as follows:

\section{The data misfit function $\phi^{d}$}

The amplitude of marine CSEM data spans several orders of magnitude, therefore it is important to work with some form of data misfit function that promotes a relatively uniform fit throughout the data set. Here, the function used is the relative difference between the calculated and the observed data: in each iteration, the calculated data are normalized by the corresponding observations, and the inversion will try to fit these ratios to the constant value of 1 (Almeida \& Rijo, 2011).

The functional $\phi^{d}(\mathbf{m})$ is represented as

$$
\phi^{d}(\mathbf{m})=\|(\overline{\mathbf{0}}-\overline{\mathbf{r}}(\mathbf{m}))\|_{2},
$$

where $\overline{\mathbf{0}}$ is a vector whose components are all ones and $\overline{\mathbf{r}}(\mathbf{m})$ is the vector whose components are the ratios between calculated data $\left(y^{\text {calc }}\right)$ and the observations $\left(y^{o b s}\right)$ :

$$
\overline{\mathbf{0}}=\left[\begin{array}{c}
1 \\
1 \\
\vdots \\
1
\end{array}\right] ; \quad \overline{\mathbf{r}}(\mathbf{m})=\left[\begin{array}{c}
\mathrm{r}_{1} \\
\mathrm{r}_{2} \\
\vdots \\
\mathrm{r}_{N}
\end{array}\right] ; \quad \mathrm{r}_{i}=\frac{y_{i}^{\text {calc }}}{y_{i}^{\text {obs }}} .
$$

This definition for the data misfit function has the consequence that the entries in each row of the sensitivity matrix will also be normalized by the respective observation.

\section{The model objective function $\phi^{p}$}

The regularizing function $\phi^{p}(\mathbf{m})$ through which the method introduces a priori information is composed of two parts:

$$
\phi^{p}(\mathbf{m})=\alpha \phi^{R E}+\gamma \phi^{T V} .
$$

The functions $\phi^{R E}$ and $\phi^{T V}$ are defined to introduce the two types of constraints used here. The regularizing parameters $\alpha$ and $\gamma$ are used to weigh the influence of the a priori information relatively to the data. These parameters should be as small as possible, while still yielding a stable solution.

\section{The Relative Equality constraint $\phi^{R E}$}

The function $\phi^{R E}$ is used to impose relative equality constraints (Medeiros \& Silva, 1996) between the two conductivity values in each layer of the interpretative model. The motivation for this is to cope with the fact that the CSEM data have quite distinct sensitivities to the two different conductivities in anisotropic layers.

A thorough analysis of how the two components of the anisotropy affect differently the components of the measured electric field is presented in Ramananjaona et al. (2011). As the electromagnetic field diffuses through the layered medium, we can think of it, mathematically, as the composition of two propagation modes: the transverse electric (TE) mode, in which the electric field lines are only on the horizontal direction and are, therefore, affected only by the horizontal conductivity; and the transverse magnetic (TM) mode, in which the electric field lines cross the layers in vertical planes, so that they are affected by both conductivity components. The measured inline electric field component $\left(E_{x}\right)$ is mostly affected by the TM mode field, so it is influenced by both vertical and horizontal conductivities, their relative influence being dependent on geometric parameters, like the layered structure and the source-receiver offset, as well as on the frequency. On the other hand, the measured broadside electric field component $\left(E_{x}\right.$, at an azimuth of $90^{\circ}$ in relation to the dipole orientation) is strongly affected by the TE mode field, therefore it is more influenced by the horizontal conductivity. The TM mode propagation is influenced by both inductive and galvanic effects, as the current lines cross the interfaces between layers. Propagation in the TE mode is made only of inductive effects, with the current lines always tangent to the interfaces. This has an important implication to the case of marine CSEM, where most of the medium is highly conductive and the target layers are comparatively much more resistive. In this case, the influence of a resistive layer is felt more strongly in the component that is sensitive to galvanic effects than in the one that is more influenced by inductive effects. Furthermore, the inductive influence of the resistive layer will be week, compared with that of the surrounding medium. All these effects contribute to the fact that the vertical conductivity influences the data much strongly than the horizontal conductivity, which implies a much better resolution of vertical conductivities by the inversion process. 
In order to allow the better detected vertical conductivity $\left(\sigma_{v}\right)$ to influence the resolution of the horizontal one $\left(\sigma_{h}\right)$, the relative equality constraint $\left(\phi^{R E}\right)$ will be applied to the components in each layer by making

$$
\phi^{R E}=\|\mathbf{D} \mathbf{m}\|_{2},
$$

where $\mathbf{D}$ is the matrix whose rows are filled with zeroes, except for two positions, corresponding to two parameters whose values are to be close to each other. The elements of $\mathbf{D}$ in these positions will be 1 and -1 . For instance, for the $i^{t h}$ and $j^{t h}$ parameters, the matrix will have a row of the form $\left[\begin{array}{llllllll}0 & 0 & \cdots & 1 & -1 & \cdots & 0 & 0\end{array}\right]$ where the numbers 1 and -1 are at the $i$-th and $j$-th positions, respectively.

The parameters $m_{1}$ and $m_{2}$ are the logarithms of the two conductivities of the first layer; $m_{3}$ and $m_{4}$ are the values of the second layer, and so on. Then for $N$ layers, the function $\phi^{R E}$ represents the equations

$$
\mathbf{D} \mathbf{m}=0,
$$

$$
\left[\begin{array}{ccccccccc}
1 & -1 & 0 & 0 & 0 & 0 & \cdots & 0 & 0 \\
0 & 0 & 1 & -1 & 0 & 0 & \cdots & 0 & 0 \\
0 & 0 & 0 & 0 & 1 & -1 & \cdots & 0 & 0 \\
& & & & & & \ddots & & \\
0 & 0 & 0 & 0 & 0 & 0 & \cdots & 1 & -1
\end{array}\right]\left[\begin{array}{c}
m_{1} \\
m_{2} \\
m_{3} \\
\vdots \\
m_{2 N}
\end{array}\right]=\left[\begin{array}{c}
0 \\
0 \\
0 \\
\vdots \\
0
\end{array}\right],
$$

and the equalities are imposed in the least squares sense.

The incorporation of these equality constraints to the conductivities of each layer forces them to change in tandem and prevents the horizontal components from converging to geologically meaningless values. It also helps to achieve the right convergence to the parameters associated with isotropic layers. This has also been found to be true in the inversion of magnetotelluric data from anisotropic media (Régis et al., 2010).

\section{The Total Variation constraint $\phi^{T V}$}

The function $\phi^{T V}$ is used to introduce the Total Variation (TV) constraint, to allow for abrupt changes in the parameters. This function enforces equality between the same conductivity components in every two adjacent layers. For the layers $(i)$ and $(i+1)$, the relations expressed by this function are

$$
\begin{aligned}
& \log _{10} \sigma_{h}^{(i)}-\log _{10} \sigma_{h}^{(i+1)}=0, \\
& \log _{10} \sigma_{v}^{(i)}-\log _{10} \sigma_{v}^{(i+1)}=0 .
\end{aligned}
$$

These are incorporated in the stabilizing function through the matrix $\mathbf{S}$,

$$
\mathbf{S} \mathbf{m}=0,
$$

$$
\left[\begin{array}{ccccccc}
1 & 0 & -1 & 0 & 0 & 0 & \cdots \\
0 & 1 & 0 & -1 & 0 & 0 & \cdots \\
0 & 0 & 1 & 0 & -1 & 0 & \cdots \\
0 & 0 & 0 & 1 & 0 & -1 & \cdots \\
& & & & & & \ddots
\end{array}\right]\left[\begin{array}{c}
m_{1} \\
m_{2} \\
m_{3} \\
\vdots \\
m_{2 N}
\end{array}\right]=\left[\begin{array}{c}
0 \\
0 \\
0 \\
\vdots \\
0
\end{array}\right],
$$

but now the deviations from the equalities are measured in the $L_{1}$ norm $\left(\|\cdot\|_{1}\right)$, instead of $L_{2}$ norm:

$$
\phi^{T V}=\|\mathbf{S} \mathbf{m}\|_{1} .
$$

According to the definition of the norm, the function $\phi^{T V}$, for $N$ layers in the interpretative model and $2 N$ parameters, is written as:

$$
\phi^{T V}=\sum_{i=1}^{N-1}\left(\left|\log _{10} \sigma_{h}^{(i)}-\log _{10} \sigma_{h}^{(i+1)}\right|+\left|\log _{10} \sigma_{v}^{(i)}-\log _{10} \sigma_{v}^{(i+1)}\right|\right) .
$$


However, this function is not differentiable whenever the differences are zero. In order to avoid this difficulty and allow the method to operate with the gradient in each iteration, the function is modified (Acar \& Vogel, 1994) by the approximation

$$
\left|\log _{10} \sigma^{(i)}-\log _{10} \sigma^{(i+1)}\right| \approx\left[\left(\log _{10} \sigma^{(i)}-\log _{10} \sigma^{(i+1)}\right)^{2}+\beta\right]^{1 / 2}
$$

where $\beta$ is a small positive number. The detailed derivation of the expressions for the gradient vector $g$ and Hessian matrix $H$ associated with the modified $\phi^{T V}$ function is presented in Martins et al. (2011), where it is shown how the value of $\beta$ affects the behaviour of these operators, with larger values generating smoother solutions. The solution is sought iteratively, using the Gauss-Newton method, with the Levenberg-Marquardt strategy (Marquardt, 1963; Pujol, 2007).

The iterative process involves taking the Taylor expansion of $\Phi^{d}(\mathbf{m})$ about the approximation $\hat{\mathbf{m}}_{k}$, which is obtained in the $k^{\text {th }}$ iteration, and keeping the terms up to second-order. Then, taking the derivative of this function, $\Phi^{d}\left(\hat{\mathbf{m}}_{k}+\Delta \mathbf{m}_{k}\right)$, with respect to $\Delta \mathbf{m}_{k}$ and setting this derivative to zero leads to the normal equation to the estimate of $\Delta \mathbf{m}_{k}$ :

$$
\mathbf{H}_{k} \Delta \mathbf{m}_{k}=-\mathbf{g}_{k} .
$$

Define $J$ as the sensitivity (Jacobian) matrix. Each entry in this matrix is the derivative of the fitting functional with respect to one of the parameters, normalized by the corresponding observed data, to be consistent with the definition of the data misfit function.

According to Martins et al. (2011) the Hessian matrix and the gradient vector of the functional $\Phi(m)$ are

$$
\mathbf{H}_{k}^{T V}=J^{T} J+\alpha D^{T} D+S^{T}(\gamma R+I) S,
$$

and

$$
\mathbf{g}_{k}^{T V}=-J^{T}(\bar{o}+\bar{r}(m))+\alpha D^{T} D m_{k}+S^{T} d,
$$

where $d$ is a vector with size $L$ equal to the number of pairs of parameters which are to be constrained, and $R$ is a diagonal matrix. At iteration $k$, the $l^{\text {th }}$ element of vector $\mathbf{d}$ and the $l^{\text {th }}$ diagonal entry in matrix $R$ are, respectively

$$
\begin{aligned}
d_{l} & =\left.\frac{m_{i}-m_{j}}{\left[\left(m_{i}-m_{j}\right)^{2}+\beta\right]^{\frac{1}{2}}}\right|_{m=m_{k}}, \\
R_{(l, l)} & =\left.\frac{\beta}{\left[\left(m_{i}-m_{j}\right)^{2}+\beta\right]^{\frac{3}{2}}}\right|_{m=m_{k}} .
\end{aligned}
$$

The $k+1^{s t}$ iteration of the Levenberg-Marquardt method will be:

$$
\begin{aligned}
m_{k+1}= & \mathbf{m}_{k}+\left[J^{T} J+\alpha D^{T} D+S^{T}(\gamma R+I) S+\lambda I\right]^{-1} \\
& \times\left[J^{T}(\bar{o}-\bar{r}(m))-\alpha D^{T} D m_{k}-S^{T} d\right]
\end{aligned}
$$

where $\mathbf{I}$ is the identity matrix and $\lambda$ is a parameter used to control the step size of the variation of vector $\mathbf{m}$, as defined by Marquardt (1963).

These iterations are repeated, changing the value of $\lambda$ as necessary, until convergence is achieved. The convergence criterion consists in reaching a minimum, i.e. a point where the gradient is zero, or very close to zero, and also fitting the data within a specified tolerance.

The method will not penalize sharp variations because, in the $L_{1}$ norm, the total measure of misfit between all pairs of adjacent parameters will be the same, regardless of how the parameters

vary: smoothly or otherwise. If the misfit is measured through the $L_{2}$ norm, the minimum will be reached by the smoothest spatial distribution of parameter values. On the other hand, the objective function must fit the observations, which will prevent the parameter distribution from being discontinuous in places where that discontinuity can not explain the data. These points are nicely illustrated in Lima et al. (2011).

The TV method still enforces proximity between adjacent 
parameters. Therefore, if the structures that generated the data are truly smooth, then the solutions will tend to present the same general behavior as those from global smoothness methods. However, if the influence of a discontinuity on the parameter distribution is felt on the data, the TV constraints allow for abrupt changes on those parameters without the resulting oscillations that inevitably result from smoothing methods. This makes the method a valid alternative when inverting CSEM data from geological environments that are generally smooth, but can have strong discontinuities in places like the interfaces between geoelectric layers, as in the border of an oil zone or of a salt body, which can be very resistive zones inside conductive sediments.

\section{RESULTS}

We show results for two different sets of synthetic data and one set of real data from which the inversion tries to identify resistive target layers. For all cases, the initial guess was a homogeneous half-space, indicated by the red lines in the figures. All results are expressed in units of resistivity $(\Omega \cdot m)$.

\section{Synthetic data}

The synthetic data were generated with the dipole source at $30 \mathrm{~m}$ from the sea floor. The observations are the horizontal components of the electric $\left(E_{x}\right)$ and magnetic $\left(H_{y}\right)$ fields on the sea bed, which are inverted simultaneously. Starting at a distance of
$500 \mathrm{~m}$ from the source, the fields were calculated at 20 observational points at $500 \mathrm{~m}$ intervals, up to a maximum of $10 \mathrm{~km}$ for the lowest frequency, on the line parallel to the dipole direction (inline configuration). Five frequencies were used: $0.125 \mathrm{~Hz}$, $0.50 \mathrm{~Hz}, 0.75 \mathrm{~Hz}, 1.00 \mathrm{~Hz}$ and $2.00 \mathrm{~Hz}$.

Stability was verified by adding different sequences of random noise to the data and evaluating the proximities among the corresponding solutions. The zero mean noise sequences have different intensities for data in different frequencies (Myer et al., 2012). In our examples, the data from lower frequencies are contaminated with higher noise levels, around $10^{-15} \mathrm{~V} / \mathrm{Am}^{2}$, whereas data from the higher frequencies are contaminated with a $10^{-16}$ $\mathrm{V} / \mathrm{Am}^{2}$ noise level. A representation of the noise in the same scale as the data is shown in Figure 1.

The interpretation model for both examples is formed by 79 horizontal homogeneous anisotropic layers with transversely isotropic conductivities $\left(\sigma_{h}, \sigma_{v}\right)$ and constant thicknesses of $25 \mathrm{~m}$ each, over a likewise anisotropic infinite basement. There are, therefore, 160 parameters to be estimated: two conductivities for each layer in the interpretative model.

As a base for comparison, we have computed the inversion of the data set produced by each model using only smoothness constraints, in an Occam's inversion approach, as described in Ramananjaona et al. (2011). We will refer to this method as "global smoothness", since its objective is to generate models with overall minimum structure.

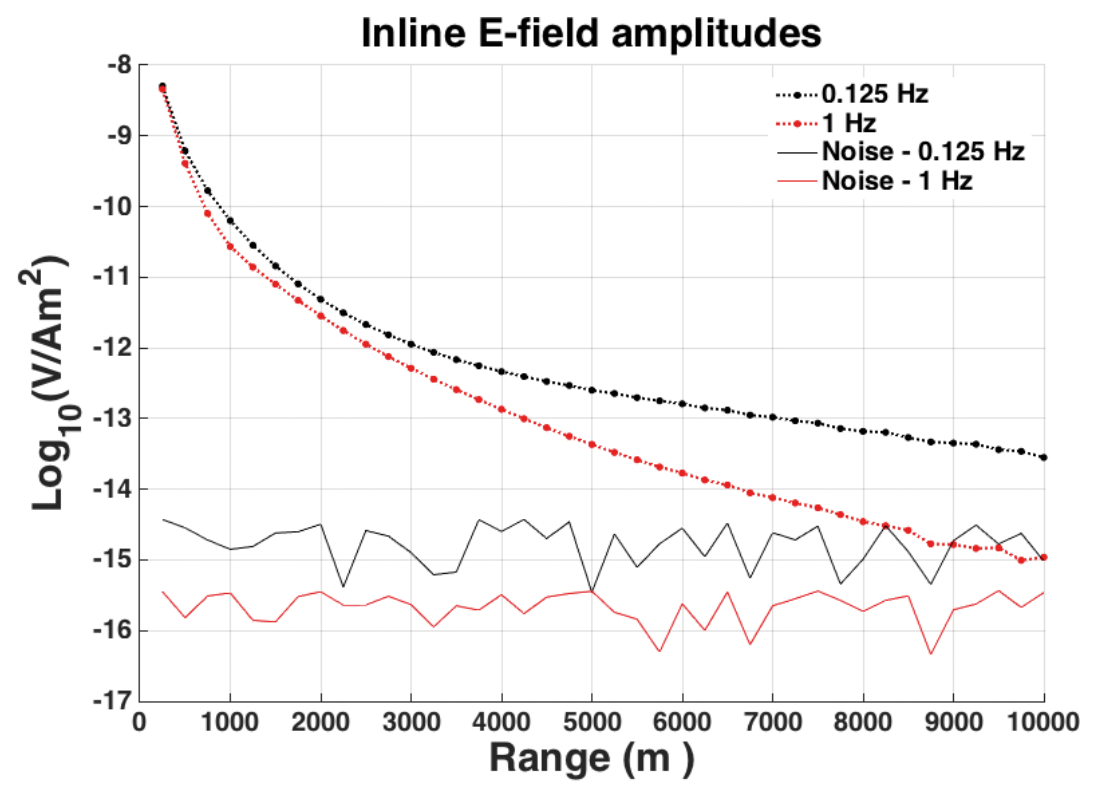

Figure 1 - Representation of the noise levels contaminating the synthetic data. The absolute values of the zero mean noise sequences are shown in the same plot as the data. 


\section{Model 1}

The first model is shown in Figure 2. Here, the resistive target layer is inside a conductive region, above a half-space basement. The water layer and the basement are isotropic.

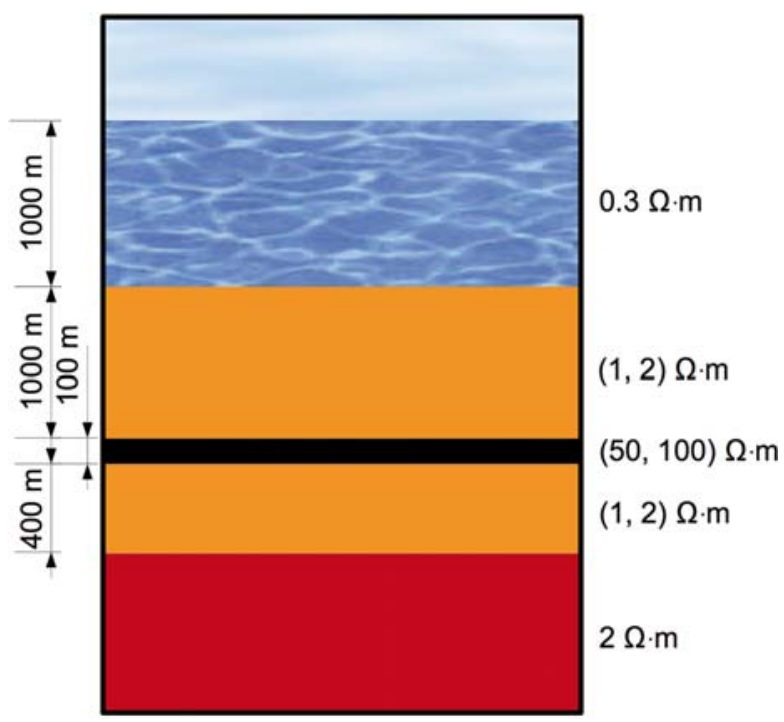

Figure 2 - First model used to generate data to be inverted.

Figure 3 shows the geometric representation of a matrix whose elements are the sensitivities of the electric field in a single receiver to variations in the two components of the conductivity in each layer at the frequency of $0.125 \mathrm{~Hz}$. Each column in these matrices represents the sensitivities for one receiver position relative to every layer of the interpretative model. The influences of the two components of the conductivity on the observations produced by the interpretation model are quite different. In all cases, the influence of the resistive layer is distinct from that of the background medium: the sensitivities to $\sigma_{v}$ inside the resistive layer are greater than the equivalent sensitivities in the background, while the sensitivities to $\sigma_{h}$ are smaller than those in the surrounding medium. The vertical conductivity imparts a stronger influence on the fields than the horizontal component because of its effect on the current flow, whereas the inductive effects that would be dominated by the horizontal component are weakened by the higher resistivity relative to that of the surrounding layers. These differences in how the fields respond to the two conductivity components imply markedly different levels of detectability, so that the vertical conductivity of the resistive layer is much better resolved than the horizontal one, as can be observed in the final results of the inversion. On the other hand, in the low resistivity parts of the model the situation is reversed: the sensitivity to the horizontal resistivity is highest.
Figure 4a shows the results obtained with the application of global smoothness only. In this case, although the solution is stable, the resulting model does not represent the true discontinuity present in the original model. By adding equality constraints between the resistivity components in each layer the process results in a better convergence to the true value of the isotropic basement, but there is no significant improvement in the delineation of the resistive layer, as seen on Figure 4b.

Figure $4 \mathrm{c}$ shows that applying the TV constraints instead of global smoothness improves the resolution of the resistive layer, by admitting a solution with sharper variation in the vertical conductivity. As a consequence, a better determination of the isotropic conductivity of the basement was achieved. Note that the horizontal component of the conductivity of the resistive layer is still undetected.

The best results are obtained with the application of the TV method with the addition of equality constraints between the two conductivities in each layer, as shown in Figure 4d. Now, the general characteristics of the true conductivity profiles are shown, especially in the vertical resistivity curve. The resistive layer is clearly identified, although the true value is approximated only by the vertical conductivity.

A remarkable difference between the two solutions is that the TV solution presents much less oscillations than the one obtained with global smoothness constraints. In the smooth solution, the effect of the sharp variation on the interfaces of the resistive layer is spread around that layer. This implies in a greater resistive zone that does not conform to the behavior of the data. Since the estimated data must fit the observations, the smooth solution tends to compensate this excess of resistive zones by generating more conducting zones next to the resistive one. This leads to models with the observed oscillations about the discontinuities of the true model. In the case of the TV solution, there are less oscillations, because the resistive zone is concentrated closer to the true resistive layer.

\section{Model 2}

The second model (Fig. 5) presents a greater challenge to the inversion. Now there are two resistive layers, which makes their delineation from the data harder. The first target layer is shallower than the one in the first model, starting at $500 \mathrm{~m}$ from the sea bed, but it has half the thickness $(50 \mathrm{~m})$ and half the resistivities of that layer. The second target layer has the same thickness and the same resistivities as that of model 1 , but it is deeper, starting at $1500 \mathrm{~m}$ from the sea bed. 

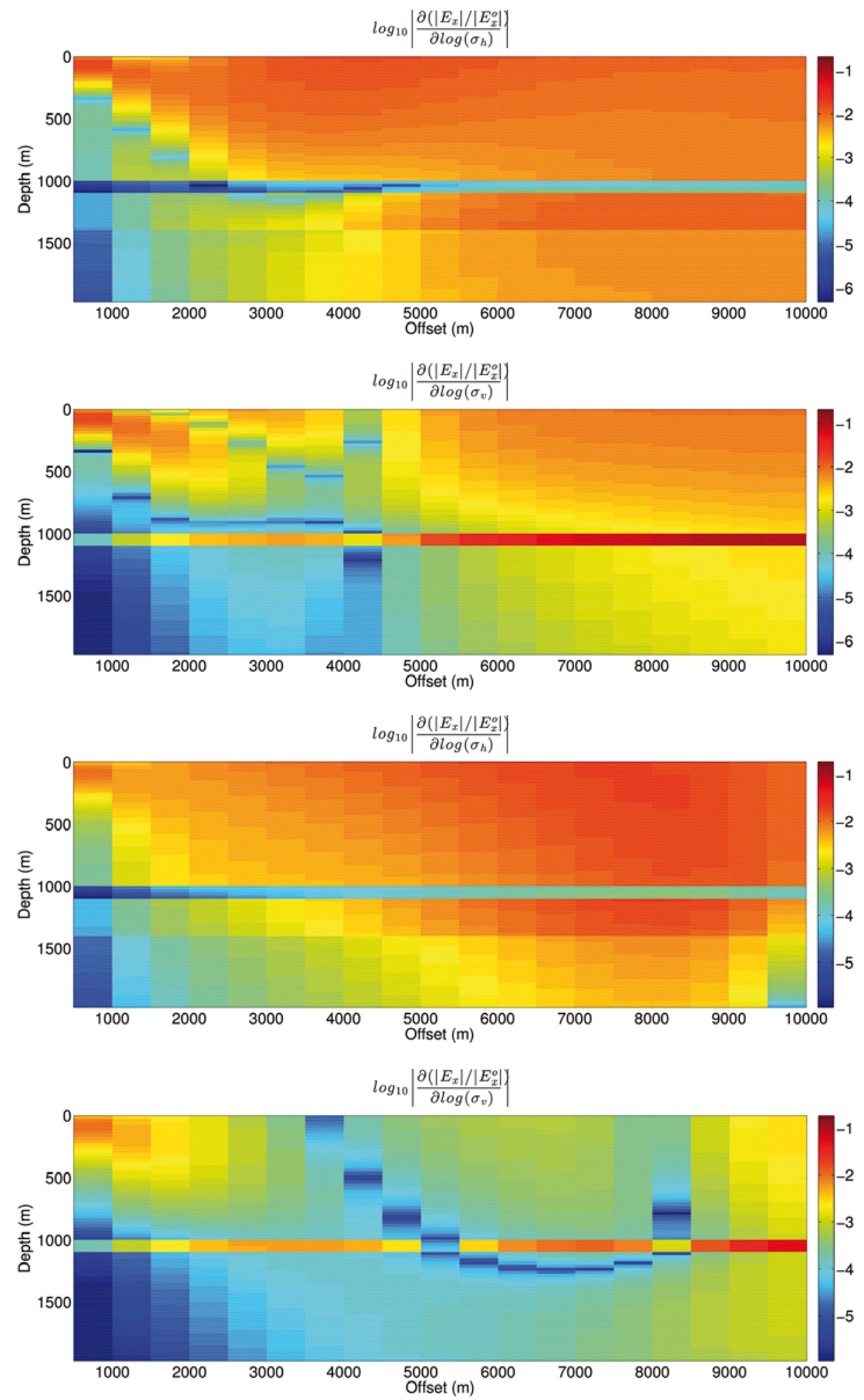

Figure 3 - Sensitivity of the electric field data from model 1 with respect to the two components of the conductivity in both inline and broadside configurations at $0.125 \mathrm{~Hz}$ 

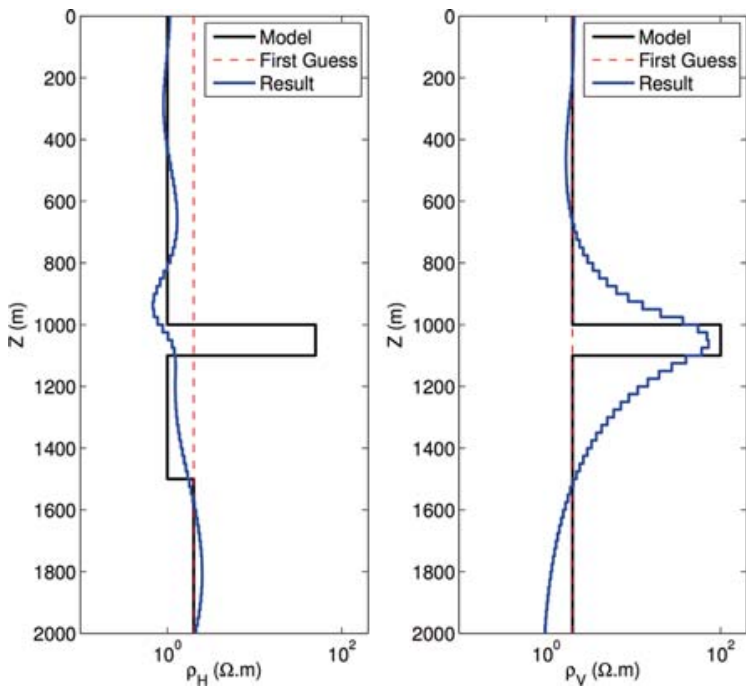

(a) - Result obtained with the application of global smoothness only.
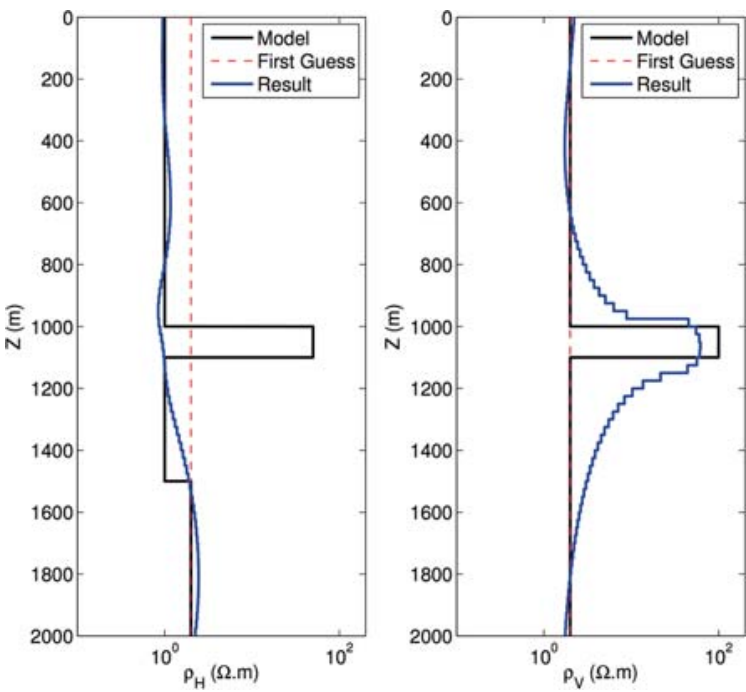

(c) - Result obtained with TV constraints only.
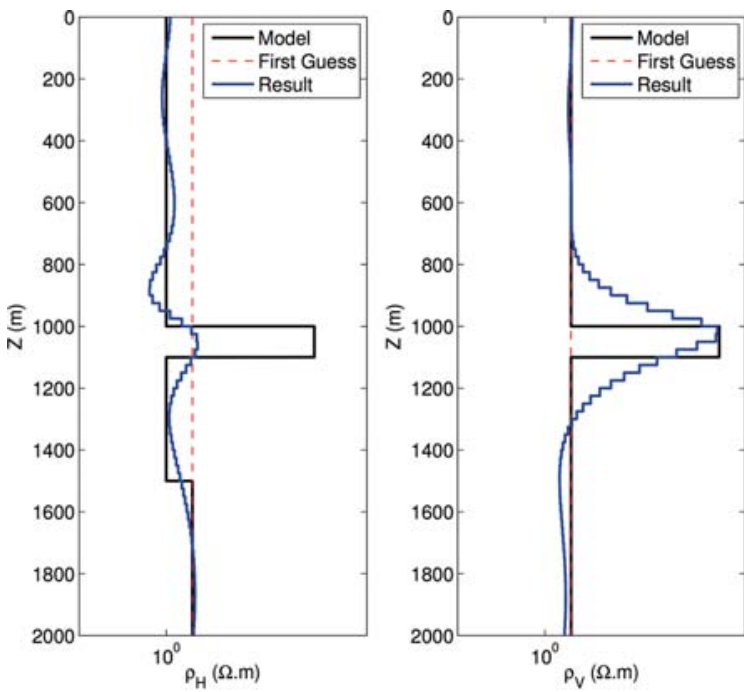

(b) - Result obtained with the application of global smoothness and equality between the anisotropic conductivity components in each layer.
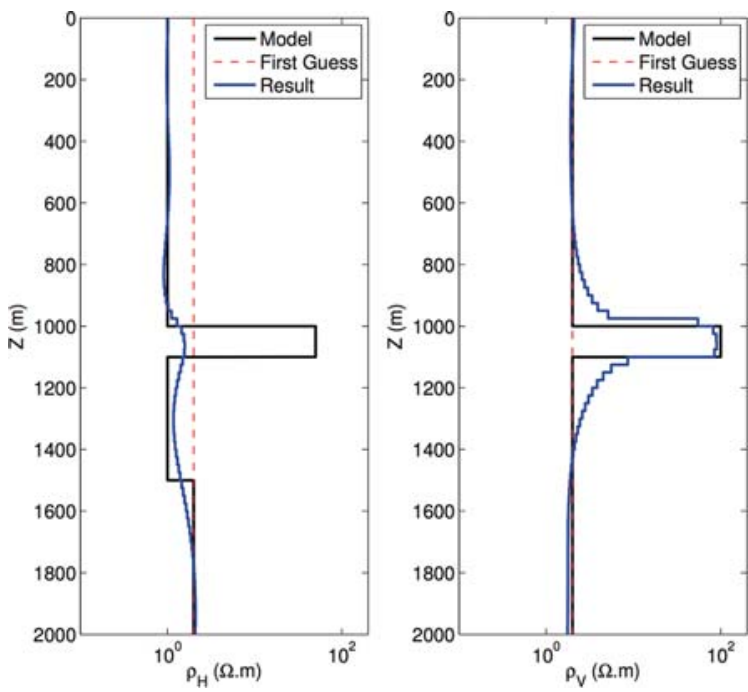

(d) - Result obtained with TV constraints and equality between the anisotropic conductivity components in each layer.

Figure 4 - Inversion results for the first model, with one resistive target layer.

The electric field sensitivities for this model are shown in Figure 6, again at the frequency of $0.125 \mathrm{~Hz}$. They have the same general characteristics found for the first model, with higher sensitivities associated with the vertical components of the anisotropic conductivity, which will also lead to better resolution of that component.

The results for this model are in Figure 7. Due to the smaller sensitivity values associated with the second layer and the simultaneous influence of the two resistive layers on the data, the delineation of the two targets is not as accurate as in the case of the first model, although the method still yields better results than the application of traditional smoothness constraints.

The horizontal components of the conductivity of the target layers are, again, practically undetected. However, the method is still able to achieve a better focus on the targets in the vertical resistivity profile, and a better estimation of the resistivities of the background medium in comparison with the Occam approach. 


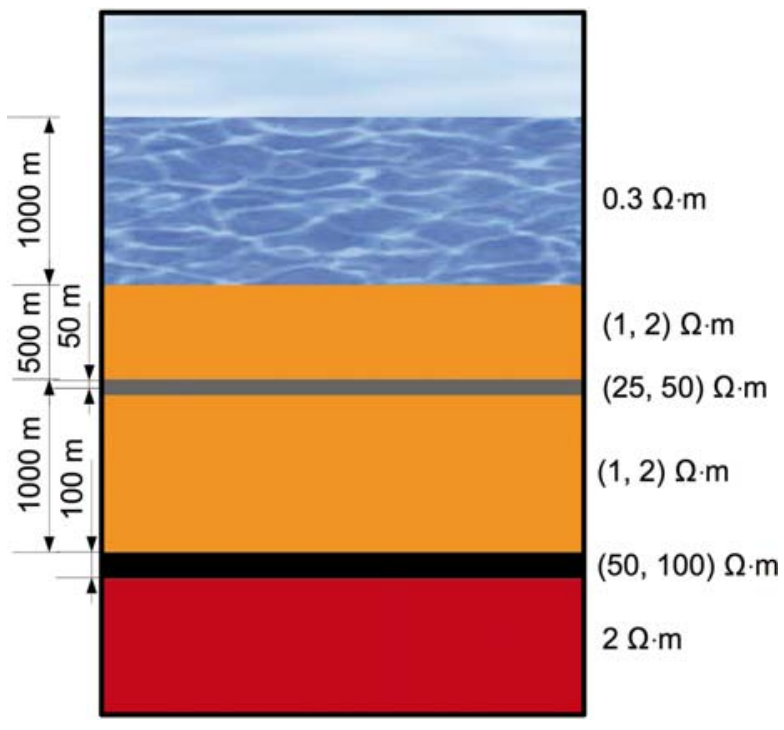

Figure $\mathbf{5}$ - Second model used to generate data to be inverted, with two target layers.

\section{Real data}

We have applied the method to a real data set acquired by EMGS in the offshore Espirito Santo basin in southeastern Brazil. The data are part of a large multiclient acquisition campaign covering several Brazilian offshore basins. The particular set to which we have applied the $1 \mathrm{D}$ inversion has already been successfully used by PETROBRAS in an interpretation workflow (Buonora et al., 2014).

The inversion was performed on the data from a single measurement position, at a southwest-northeast towline over a previously acquired seismic line. This line crosses a successful well (referred to as W1), which intercepted a $100 \mathrm{~m}$ thick hydrocarbon layer at a $3720 \mathrm{~m}$ depth, with an average resistivity of 70 ohm-m. The line is laid between two salt structures which appear prominently in the seismic section, as shown in Buonora et al. (2014). We chose to work with data from the receiver closest to the well, located approximately at $1.5 \mathrm{~km}$ from the flank of the southeast salt body.

The data set is composed of amplitudes and phases of the inline electric field at four frequencies. We have selected data only from transmitter positions to the right of the receiver, in order to reduce the influence of the $3 \mathrm{D}$ salt structure on the left. Longest transmitter-receiver offsets varied from $4,101.33 \mathrm{~m}$, at $1.406 \mathrm{~Hz}$, to $6,900.11 \mathrm{~m}$, at $0.1562 \mathrm{~Hz}$.

The data fit is illustrated in Figure 8. The curves show a comparison between the observed data and those from the model generated by the global smoothness inversion. The real data curves show variations that can not be fitted by the calculated layered model fields. However, we still find a good fit, with most amplitude points within a 10\% misfit, and even less for the phase points.

The results are illustrated in Figures 9 and 10, overlaid on a section obtained from the inversion method described by Buonora et al. (2014), which doesn't take the anisotropy of the medium into account. Two distinct behaviors can be observed in the results, in two depth zones:

1- At depths greater than about $4000 \mathrm{~m}$ the horizontal resistivities appear higher than the vertical resistivities. This nonsensical behavior results from the fact that at the lowest frequencies and biggest offsets, the 3D geometry of the medium generates data that can not be fit by a layered model data, particularly in a situation in which the horizontal resistivity has very little influence on the observations, as indicated by the sensitivity analysis in the synthetic data section. Note that even with clearly wrong horizontal resistivities in the deeper section of the model, the data fit is not worse on the largest offsets and lowest frequencies than in the other parts of the data set, as illustrated in Figure 8.

2- Above $4000 \mathrm{~m}$, the curves show a strong indication of a resistive zone, approximately in the correct depth as indicated by Buonora et al. (2014). Whereas in the previous results these resistive regions can be interpreted as being composed of two distinct zones, in our results only the horizontal resistivity curve of the global smoothness inversion shows any indication of such division.

A common result in inversion of marine CSEM data is to Iocate the resistors in positions above the true positions of the targets. This error can be reduced by constraining the responses using a priori information, for example from a well log, as the one exhibited in Figures 9 and 10. As the study shown here doesn't include any such a priori information, the results reproduce the common behavior, and the maximum resistivity in the resulting models is located at about $500 \mathrm{~m}$ above the peak values found in Buonora et al. (2014).

\section{CONCLUSION}

The presented method proposes the simultaneous use of two kinds of constraints to the model parameters in the inversion of marine CSEM data from anisotropic layered media: one is that of the Total Variation method, defined as L1 norm equality constraints, and the other is the enforcing of equality on the two components of the conductivity in each layer.

The TV method improved the results by allowing sharp variations, while still enforcing equality between adjacent parameters 

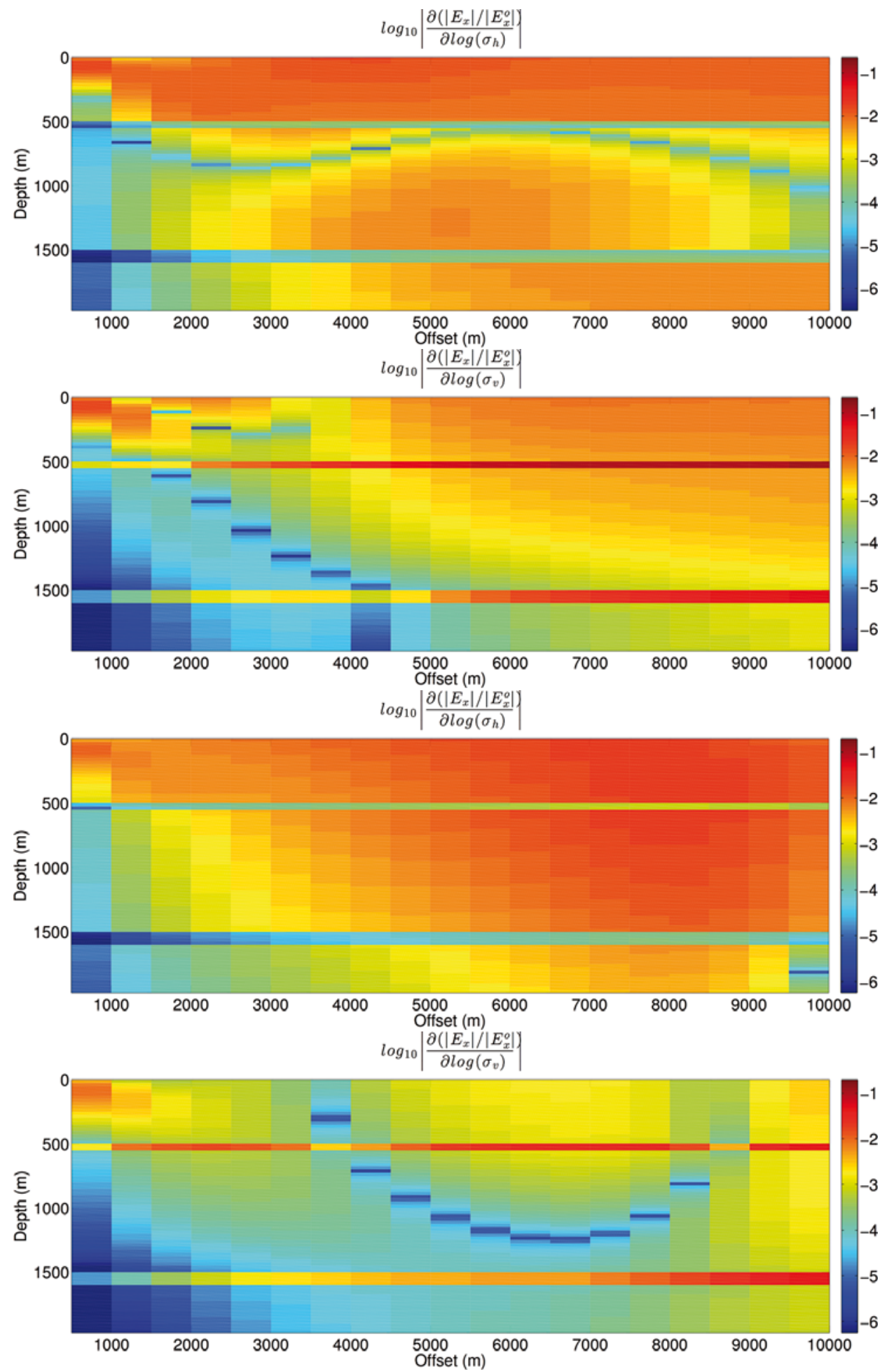

Figure 6 - Sensitivity of the electric field data from model 2 with respect to the two components of the conductivity in both inline and broadside configurations at $0.125 \mathrm{~Hz}$ 

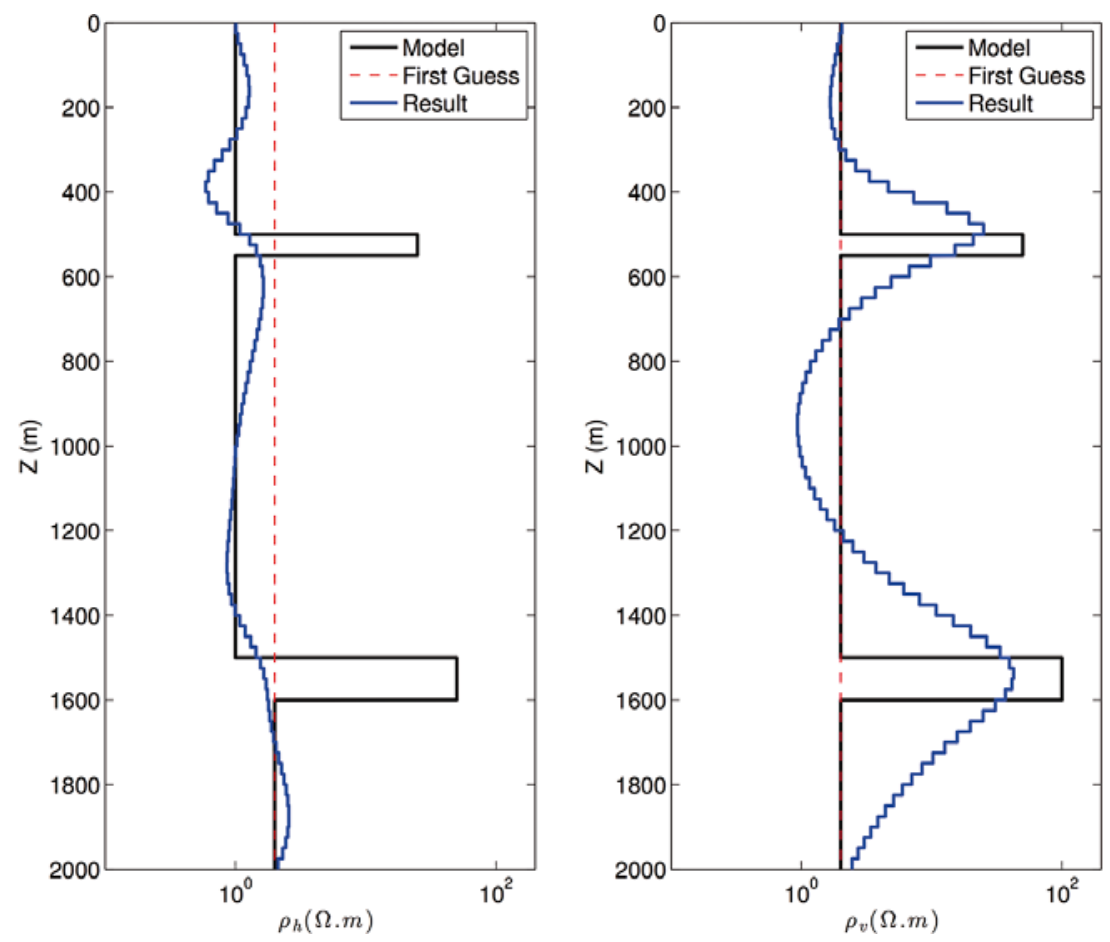

(a) - Global smoothness.
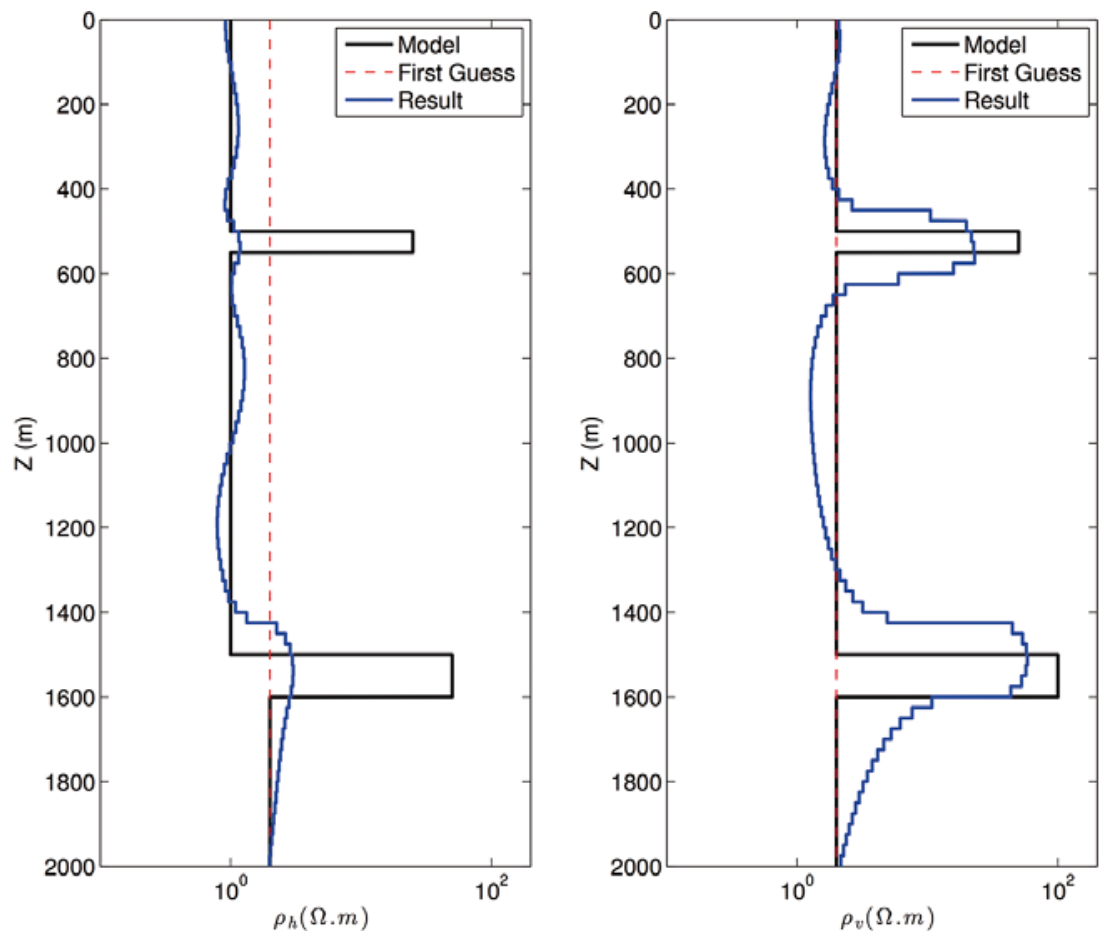

(b) - TV constraints and equality between the anisotropic conductivity components in each layer.

Figure 7 - Inversion results for the second model, with two resistive target layers. 

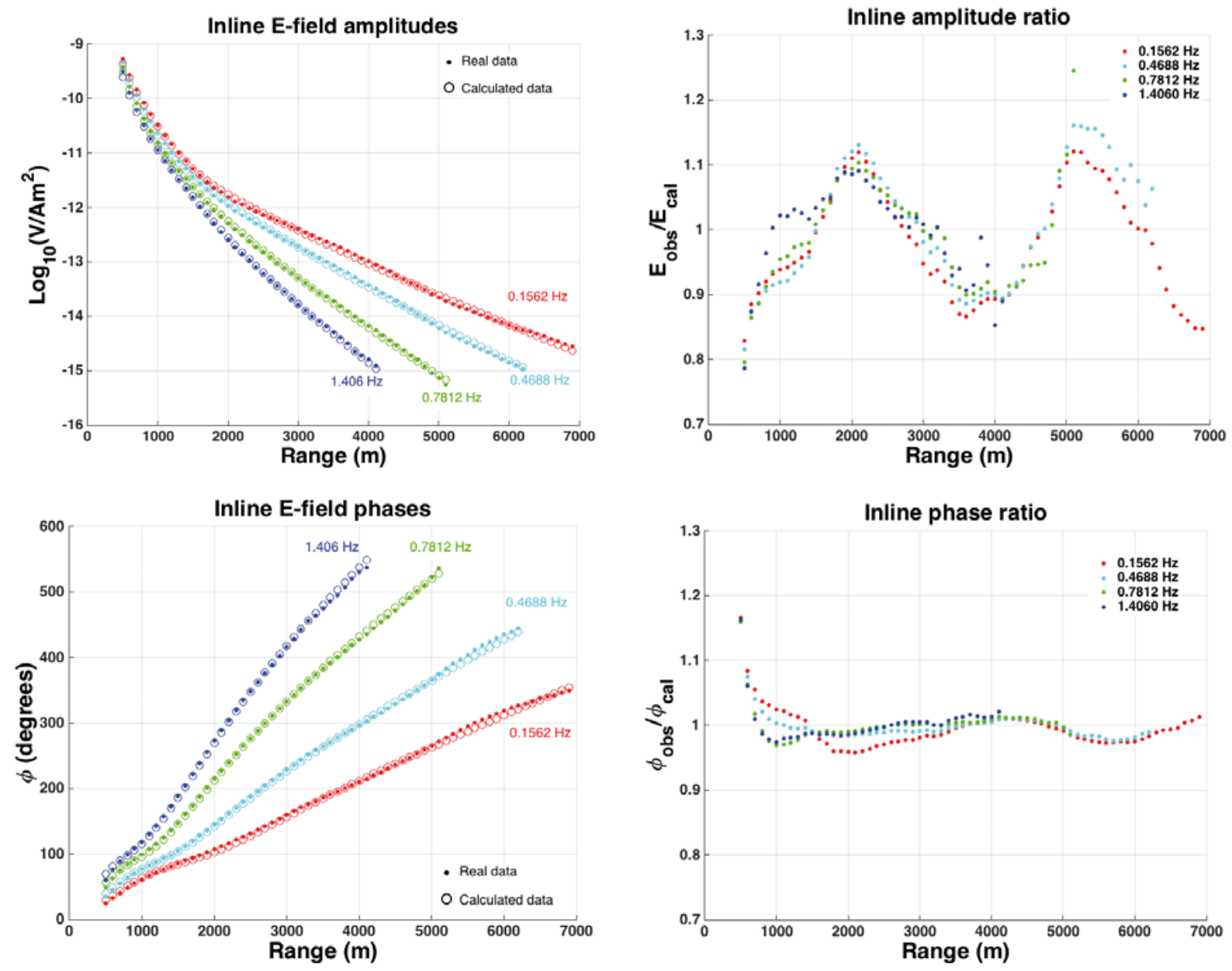

Figure 8 - Comparison between observed and calculated data sets.

wherever that is required by the fitting of the observations. The addition of equality constraints to the components of the conductivity in each layer improves resolution of the anisotropic layers by enforcing that both profiles be affected by the same discontinuities. By demanding proximity between the two components, they also help convergence to the correct resistivity of isotropic layers. Each of these two constraints has a decisive effect that complements the other, in a synergetic way, to find the solutions. When applied alone, neither is capable of generating solutions that are both stable and close to the true solution, but their combined effects are able to drive the inversion process to the sought result.

A reliable estimation of the horizontal component of the anisotropic conductivity is still an unmet challenge because of the poor sensitivity of the data with respect to that component. However, the application of the presented method resulted in better identification and delineation of the resistive targets in $1 \mathrm{D}$ problems, in comparison with the application of smoothness constraints in the traditional way.

\section{ACKNOWLEDGEMENTS}

Special thanks to professor João Batista Correia da Silva, for his critical reading of the manuscript and excellent suggestions to improve it. PETROBRAS, supported this work through the research project n. 0050.0046724.08.9. The authors thank the National Institute of Science and Technology of Petroleum Geophysics - INCT/GP. Luz thanks the PRH-06 program of the National Petroleum Agency - ANP, and Santos thanks the National Council of Scientific and Technological Development $\mathrm{CNPq}$, for their scholarships. Régis is recipient of the CNPq Research Grant.

\section{REFERENCES}

ACAR R \& VOGEL CR. 1994. Analysis of bounded variation penalty methods for ill-posed problems. Inverse Problems, 10: 1217-1229.

ALMEIDA FL \& RIJO L. 2011. Modelagens direta e inversa de dados mCSEM 3D. Brazilian Journal of Geophysics, 29(2): 289-308. 

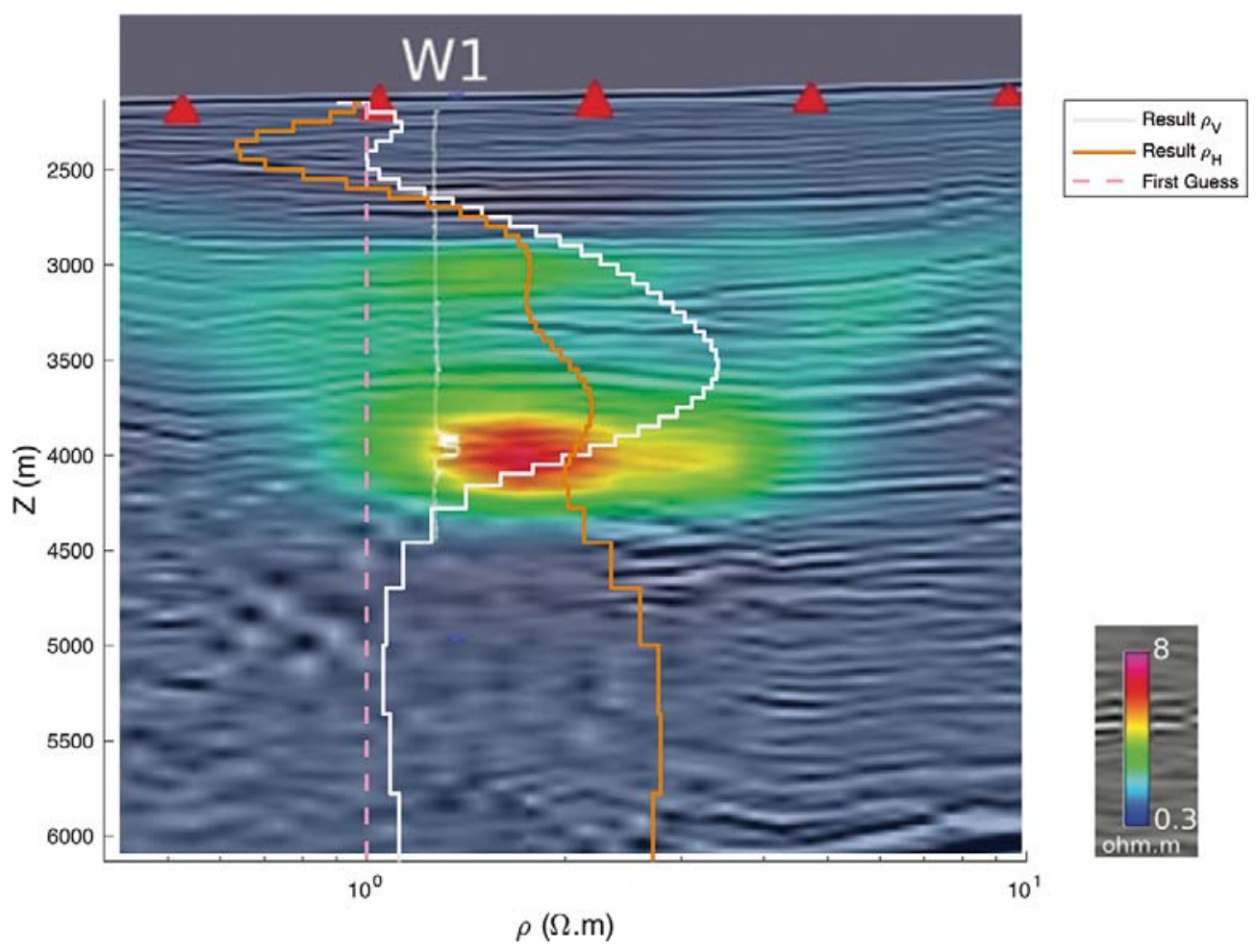

Figure $\mathbf{9}$ - Real data example: results with global smoothness constraints.

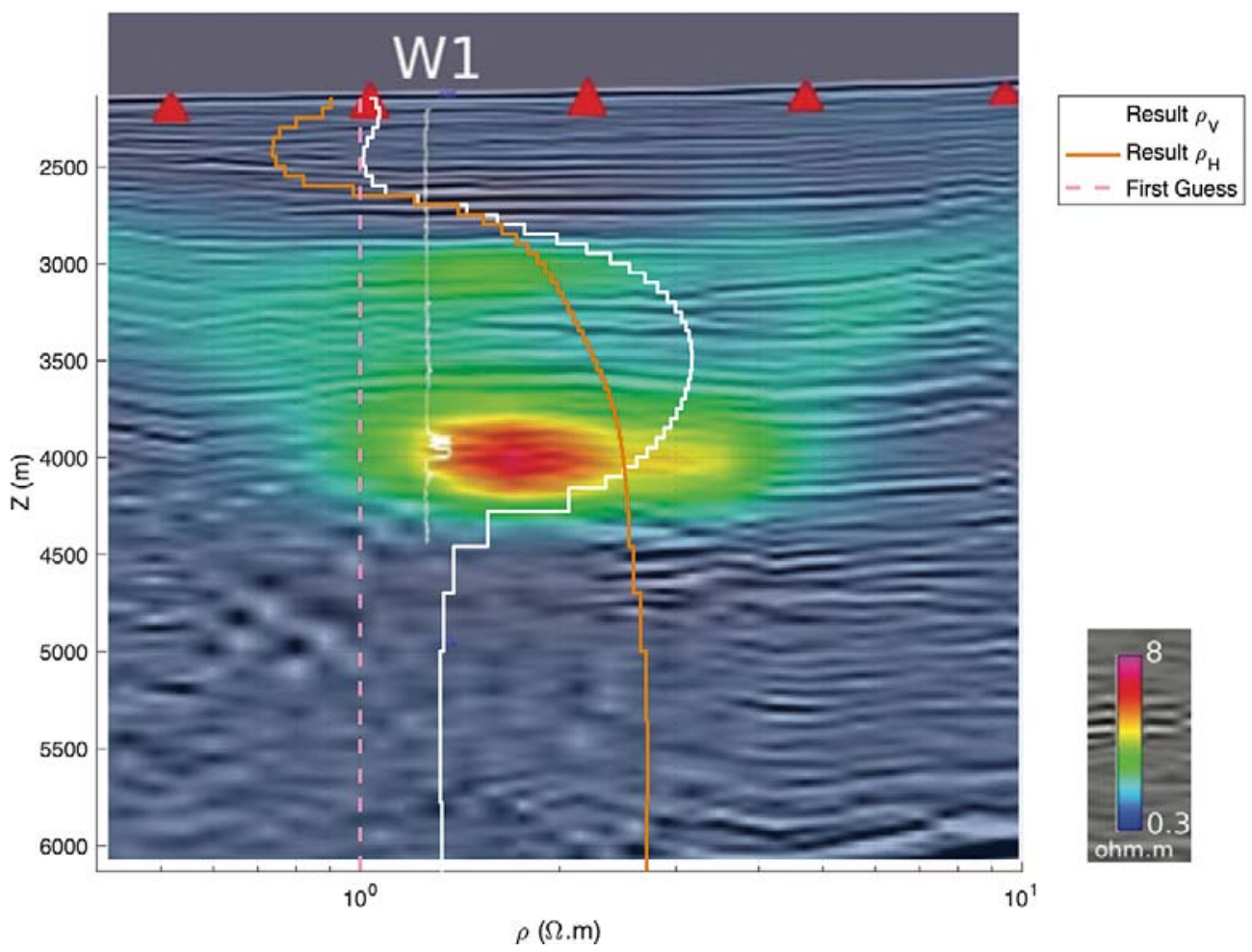

Figure $\mathbf{1 0}$ - Real data example: results with Total Variation constraints. 
ANDERSON WL. 1979. Numerical integration of related Hankel transforms of orders 0 and 1 by adaptive digital filtering. Geophysics, 44: $1287-1305$

ARFKEN G. 1985. Mathematical methods for physicists, 3rd ed., Academic Press, San Diego, 985 pp.

BUONORA MPP, CORREA JL, MARTINS LS, MENEZES PTL, PINHO EJC, CREPALDI JLS, RIBAS MPP, FERREIRA SM \& FREITAS RC. 2014. mCSEM data interpretation for hydrocarbon exploration: A fast interpretation workflow for drilling decision. Interpretation, 2: SH1-SH11.

CONSTABLE SC, PARKER RL \& CONSTABLECG. 1987. Occam's inversion: a practical algorithm for generating smooth models from electromagnetic sounding data. Geophysics, 52: 289-300.

CREPALDI JLS, BUONORA MPP \& FIGUEIREDO I. 2011. Fast marine CSEM inversion in the CMP domain using analytical derivatives. Geophysics, 76: F303-F313.

FARQUHARSON CG \& OLDENBURG DW. 1998. Non-linear inversion using general measures of data misfit and model structure. Geophys. J. Int., 134: 213-227.

KAUFMAN A \& DASHEVSKY YA. 2003. Principles of induction logging, chapter 11: The influence of anisotropy on the field of the magnetic dipole in a conducting medium. Methods in Geochemistry and Geophysics, 38: 605-625, Elsevier.

KEY K. 2009. 1D inversion of multicomponent, multifrequency marine CSEM data: Methodology and synthetic studies for resolving thin resistive layers. Geophysics, 74: F9-F20.

LIMA WA, MARTINS CM, SILVA JBC \& BARBOSA VCF. 2011. Total variation regularization for depth-to-basement estimate: Part 2 - physicogeologic meaning and comparisons with previous inversion methods. Geophysics, 76: 113-120.

MARQUARDT DW. 1963. An algorithm for least-squares estimation of nonlinear parameters. SIAM Journal on Applied Mathematics, 11: 431441.
MARTINS CM, LIMA WA, BARBOSA VCF \& SILVA JBC. 2011. Total variation regularization for depth-to-basement estimate: Part 1 - mathematical details and applications. Geophysics, 76: 11-112.

MEDEIROSWE \& SILVA JBC. 1996. Geophysical inversion using aproximate equality constraints. Geophysics, 61: 1678-1688.

MYER D, CONSTABLE S, KEY K, GLINSKY ME \& LIU G. 2012. Marine CSEM of the Scarborough gas field, Part 1: Experimental design and data uncertainty. Geophysics, 77: E281-E299.

PUJOL J. 2007. The solution of nonlinear inverse problems and the Levenberg-Marquardtmethod. Geophysics, 72: W1-W16.

RAMANANJAONA C, MacGREGOR L \& ANDRÉIS D. 2011. Sensitivity and inversion of marine electromagnetic data in a vertically anisotropic stratified earth. Geophysical Prospecting, 59: 341-360.

RÉGIS C, LUZ EC \& COSTA MD. 2010. Inversion of anisotropic MT data using approximate equality constrains. SEG Technical Program Expanded Abstracts, 29: 900-904.

RUDIN LI, OSHER S \& FATEMI E. 1992. Nonlinear total variation based noise removal algorithms. Physica D, 60: 259-268.

SANTOS WG \& RÉGIS CRT. 2015. A study of the electromagnetic field from the MCSEM dipole source in an anisotropic layered earth. Brazilian Journal of Geophysics, 33(2): 277-294.

SCHWARZBACH C \& HABER E. 2013. Finite element based inversion for time-harmonic electromagnetic problems. Geophysical Journal International, 193: 615-634.

TARANTOLA A. 2005. Inverse problem theory and methods for model parameter estimation. Society for Industrial and Applied Mathematics, Philadelphia, $348 \mathrm{pp}$.

VOGEL CR \& OMAN ME. 1998. Fast, robust total variation-based reconstruction of noisy, blurred images. IEEE Trans. Img. Proc., 7: 813-824. 